\title{
The effect of ultrasonic energy on the enhancement of the biodegradability of food waste
}

\begin{abstract}
In this study, the effect of ultrasonic pre treatment on the physicochemical property changes and methane production potential of anaerobic digestion of food waste was investigated. The study, involving a laboratory experimentation by sonicating $200 \mathrm{~mL}$ of food waste sample, was investigated at different sonication times $(2,4,6,8,10 \mathrm{~min})$, and the specific energy input ranged from 5,396 to $25,997 \mathrm{~kJ} / \mathrm{kg}$ total solid. The experimental results found that the mean particle size diameter (d50) of the ultrasonically treated food waste decreased from 59 to $21.9 \mu \mathrm{m}$ with the specific surface area increasing from 0.523 to $1.2 \mu \mathrm{m} 2 / \mathrm{g}$. This is evidenced in the food waste morphological structure changes from particles or granules varying in size and shape from small to large and round to oval or unsymmetrical shaped when the food waste samples were sonicated in the range of 2-10 min as shown by scanning electron microscopy. The value of soluble chemical oxygen demand was increased about 34$40 \%$ while the degree of disintegration was recorded as $57.15,61.10,71.08,68.94$, and $68.68 \%$, respectively. The CODsolubilization was achieved around 11.4, 11.8, 13.4, 12.7, and $13.2 \%$, when the food waste sample was sonicated at 2, 4, 6, 8, and $10 \mathrm{~min}$, respectively. This COD solubilization correlated well with DD when the linear relationship was shown by R2 = 0.945. The result shows that the use of sonicated food waste in the anaerobic digestion process has increased about $45.9,40.3,46.6,64.4$, and $70.5 \%$ of cumulative $\mathrm{CH} 4$ production rates in the food waste sample sonicated at 2, 4, 6, 8, and $10 \mathrm{~min}$, respectively, when compared to the non-sonicated food waste sample.
\end{abstract}

Keyword: Anaerobic digestion; Chemical oxygen demand solubilization; Degree of disintegration; Food waste; Ultrasonic 